\title{
FIXAÇÃO BIOLÓGICA DE NITROGÊNIO EM PASTAGENS COM DIFERENTES INTENSIDADES DE CORTE*
}

\author{
BIOLOGICAL NITROGEN FIXATION IN GRASSLAND WITH DIFFERENT CUTTING \\ INTENSITIES
}

Silva, L.L.G.G. ${ }^{1}$, Alves, G.C. ${ }^{2 A}$, Ribeiro, J.R.A. ${ }^{2 B}$, Urquiaga, S. ${ }^{2 \mathrm{C}}$, Souto, S.M. ${ }^{2 \mathrm{D}}$, Figueiredo, M.V.B. ${ }^{3}$ e Burity, H.A. ${ }^{4}$

${ }^{1}$ UFRRJ. Coordenação da Área Verde. IPJBRJ. Rua Jardim Botânico, 1008. CEP 22470180. Rio de Janeiro RJ. Ilggalindo@yahoo.com.br

${ }^{2}$ UFRRJ. Embrapa. Agrobiologia. BR 465, km 47. CEP 23851970. Seropédica RJ. Brasil. Agabrielacalves@yahoo.com.br; Bjabetous@yahoo.com.br; Csegundo@cnpabembrapa.br; Dsmsoouto@cnpab.embrapa.br

${ }^{3}$ EPEAL. IPA. CP 699. CEP 57025050. Maceió, AL. Brasil. mbarreto@elogica.com.br

4Embrapa. IPA. CP 1022. CEP50761000. Recife, PE. Brasil. burity@ipa.br

\section{PalaVRas chaVe adicionaIs}

Brachiaria. Capim elefante. Bactérias diazotróficas.

\section{RESUMO}

O manejo correto numa pastagem e o uso da fixação biológica de nitrogênio (FBN) ajudam a torná-la sustentável. Foram realizados três experimentos, cada um com uma espécie de capim forrageiro na estação experimental da Empresa Pernambucana de Pesquisa Agropecuária em Itambé, na Zona da Mata de Pernambuco. Foram avaliadas a produção de matéria seca, acúmulo de nitrogênio e FBN das plantas sob vários regimes de corte. O delineamento experimental foi inteiramente casualizado com 5 repetições que foram usasadas. Para Brachiaria decumbens e $B$. humidicola foram usadas intensidades de corte 5 , $15,25 \mathrm{~cm}$ e $25 \mathrm{~cm}+80 \mathrm{~kg} \mathrm{~N} \mathrm{ha}^{-1}$, e para o Pennisetum purpureum, $5,25,50 \mathrm{~cm}$ e $50 \mathrm{~cm}+80$ $\mathrm{kg} \mathrm{N} \mathrm{ha}^{-1}$. Os períodos avaliados foram transição seca/águas e águas em 2001. A FBN foi estimada pela abundância natural $\left(\delta^{15} \mathrm{~N}\right)$. Corte drástico $(5$ $\mathrm{cm}$ ), no período de transição seca/águas, contribuiu para produção e acúmulo de nitrogênio na $B$. decumbens, mas não houve influência significativa das intensidades de cortes na FBN nas três

*Parte da dissertação do primeiro autor apresentada ao curso de Pós Graduação em Zootecnia, Departamento de Zootecnia da UFRPE.

\author{
AdDitional KEYWORDS \\ Brachiaria. Elephant grass. Diazotrophic bacte- \\ ria.
}

pastagens e nas estações. Concluiu-se que a contribuição da FBN variou com a estação e que cortes altos não teve influência sobre o $\mathrm{N}$ acumulado, deste modo confirma-se que as três gramíneas são tolerantes a cortes drásticos.

\section{SUMMARY}

The correct management of pastures and the use of biological nitrogen fixation (BNF) therein should be sustainable. As part of an investigation into how best to use BNF in pastures three experiments were performed at the experimental station of Empresa Pernambucana de Pesquisa Agropecuária, Itambé, Pernambuco. These experiments evaluated the production of dry matter and $\mathrm{N}$ accumulation and $\mathrm{BNF}$ under various cutting regimes. A random experimental design with 5 replications was used. For Brachiaria decumbens and $B$. humidicola the treatments consisted of cutting intensities of 5,15 and $25 \mathrm{~cm}$, and $25 \mathrm{~cm}$ $+80 \mathrm{~kg} \mathrm{~N} \mathrm{ha}^{-1}$, and for Pennisetum purpureum cutting intensities 5,25 and $50 \mathrm{~cm}$ and $50 \mathrm{~cm}+80$ $\mathrm{kg} \mathrm{N} \mathrm{ha}^{-1}$. The periods in which the pastures were evaluated were the transition of the dry/wet season and the wet season of 2001. Accumulation 
of $\mathrm{N}$ via BNF was estimated by the natural abundance $\left(\delta^{15} \mathrm{~N}\right)$ method. Drastic cutting $(5 \mathrm{~cm})$ in the dry/wet season transition resulted in high accumulation of dry matter and $\mathrm{N}$ in $\mathrm{B}$. decumbens, but there was no effect of cutting intensity on BNF by any of the grasses in either of the seasons. The conclusion is that the BNF contribution varied with season, and that high cutting did not have any influence on $\mathrm{N}$ accumulation, thus confirming that the three pasture grasses are tolerant to drastic cutting.

\section{INTRODUÇÃO}

O Brasil apresenta aproximadamente 200 milhões de hectares de pastagens cultivadas principalmente com gramíneas do gênero Brachiaria (Boddey et al., 2006), em função de sua maior tolerância às condições de solos ácidos e de baixa fertilidade, aliada ao seu valor forrageiro, sendo que as espécies de maior importância são a $B$. decumbens, $B$. brizantha, $B$. ruziziensis e $B$. humidicola (Sobrinho et al., 2005). Nessas áreas são manejados rebanhos bovinos num sistema extensivo, no qual baixos índices de produtividade por área são encontrados, promovendo o subpastejo e superpastejo, que contribui para a degradação de extensas áreas de pastagens em todo território nacional. Nas pastagens em 18 municípios no estado do Rio de Janeiro, incluindo as regiões norte, noroeste, serrana, litorânea, centro-sul e sul, as gramíneas de maiores ocorrências foram Brachiaria spp. (90\%) e Paspalum spp (10\%), segundo Souto et al. (2003 e 2004). Outro gênero bastante utilizado como forrageira é o Pennisetum, destinado tanto ao pastejo quanto a formação de capineiras apresentando elevada produção de biomassa (Fagundes et al., 2005).

A disponibilidade de nitrogênio no solo é, freqüentemente, limitante para o crescimento das plantas e à produtividade das culturas (Döbereiner, 1992). Quando adicionado aos solos há perdas por volatilização e lixiviação, tornando essa prática bastante onerosa (Souto, 1982).
Como os fertilizantes nitrogenados oneram os custos de produção, e a demanda de alimentos cresce ano a ano, tem-se enfatizado a necessidade de maior exploração do potencial da fixação biológica de nitrogênio atmosférico (FBN) em gramíneas tropicais, como uma saída sustentável para este impasse (Souto, 1982).

A FBN, um dos processos naturais de grande importância para a agricultura é realizada por diferentes grupos filogenéticos de microrganismos procariotos (Reis et al., 2006) comumente conhecidos como bactérias diazotróficas. Houve um avanço muito significativo das pesquisas para os mais diversos aspectos da interação planta e bactérias diazotróficas (Baldani e Baldani, 2005). Como exemplo pode-se citar a associação de algumas variedades de cana-de-açúcar com bactérias diazotróficas endofíticas, Gluconacetobacter diazotrophicus e Herbaspirillum spp. (Baldani et al., 1992), a qual se acredita contribuir até com $60 \%$ do nitrogênio acumulado nas plantas (Urquiaga et al., 1992; Boddey et al., 2000).

A técnica da abundância natural de ${ }^{15} \mathrm{~N}$ $\left(\delta^{15} \mathrm{~N}\right)$ tem sido utilizada na estimativa da contribuição da FBN associada a plantas não leguminosas. Esta técnica se baseia na diferença da abundância natural do isótopo ${ }^{15} \mathrm{~N}$ entre o nitrogênio do solo e o atmosférico e é usualmente expressada em $\delta^{15} \mathrm{~N}$ relativo à composição do $\mathrm{N}$ atmosférico (Högberg, 1997).

Estudos sobre a distribuição da abundância natural de ${ }^{15} \mathrm{~N}$ nos ecossistemas terrestres revelaram que o $\mathrm{N}$-total do solo encontrado nos ecossistemas naturais de diversos tipos de vegetação, incluindo plantas arbóreas e gramíneas forrageiras, tende a ser mais enriquecido com ${ }^{15} \mathrm{~N}$ do que o $\mathrm{N}$ das camadas de serapilheira e/ou o nitrogênio contido nos tecidos de plantas crescendo tanto em regiões tropicais como em subtropicais (Högberg et al., 1996). Este fato torna viável a utilização da técnica da abundância natural de ${ }^{15} \mathrm{~N}$ em estudos da quantificação da FBN em regiões de climas

Archivos de zootecnia vol. 59, núm. 225, p. 22. 
com temperaturas mais elevadas.

A persistência de uma pastagem é definida como a manutenção da população de plantas e sua produção ao longo do tempo, levando-se em consideração as interferências estacionais e de manejo (Matthew et al., 1999). Se por um lado, existe farta informação de pesquisa científica em relação ao manejo, relacionado à influência da intensidade (ou altura) e freqüência (ou intervalo) de corte no crescimento, composição química e valor nutritivo dos capins (Lizieire et al., 1994; Tonato et al., 2007; Pena et al., 2007), por outro, esta é escassa em relação à FBN (Souto e Takahashi, 1989). A informação sobre a melhor altura do pasto vem sendo também intensamente utilizada nas pesquisas como forma de manejar a pastagem, visto que, os resultados têm mostrado que além dela ter grande relação com os efeitos nas características estruturais, morfológicas e qualitativas da pastagem, ela apresenta uma grande vantagem, que é a facilidade de ser uma técnica de fácil acesso e padronização a todos os pesquisadores e também a produtores (Souto, 1971; Perin et al., 2006).

Objetivando avaliar os efeitos de diferentes intensidades de corte, na FBN, produção de massa seca, acúmulo de nitrogênio e na população de bactérias diazotróficas em plantas de capins forrageiros, foram conduzidos três experimentos em pastagens cultivadas com Brachiaria decumbens, Pennisetum purpureum e Brachiaria humidicola.

\section{MATERIAL E MÉTODOS}

Os experimentos de campo foram instalados em pastagens formadas de Brachiaria humidicola Rendle, B. decumbens Stapf. e Pennisetum purpureum Schum., na Estação Experimental do IPA, Empresa Pernambucana de Pesquisa Agropecuária, localizada no município de Itambé, na Zona da Mata do Estado de Pernambuco. As pastagens foram formadas num Argissolo em 1983. As parcelas $\left(4 \mathrm{~m}^{2}\right)$ foram distribuídas ao acaso em cada pastagem, apresentando área útil de 1 $\mathrm{m}^{2}$, localizadas no centro de cada unidade experimental para evitar o efeito bordadura. Os experimentos foram instalados nas pastagens em setembro de 2000, onde foi realizado o corte de uniformização de cada um dos pastos nas respectivas alturas de corte. Os dados de precipitação pluviométrica da estação experimental demonstraram maiores precipitações no período de março a agosto de 2001, sendo que o período experimental foi de setembro de 2000 a agosto de 2001. Os solos das pastagens foram analisados para determinação das características químicas no Laboratório de Fertilidade do Solo do IPA-Sede no Recife (tabela I).

O delineamento experimental utilizado foi inteiramente casualizado com cinco repetições, onde foram avaliadas três intensidades de corte, e um tratamento adicional composto pelo corte mais alto e adubação nitrogenada. Os tratamentos para $B$. decumbens e $B$. humidicola foram: altura de corte de $5 \mathrm{~cm}, 15 \mathrm{~cm}, 25 \mathrm{~cm}$ e $25 \mathrm{~cm}+80 \mathrm{~kg}$ $\mathrm{N} \mathrm{ha}^{-1}$, já para o $P$. purpureum os tratamentos foram altura de corte de $5 \mathrm{~cm}, 25 \mathrm{~cm}, 50 \mathrm{~cm}$ e $50 \mathrm{~cm}+80 \mathrm{~kg} \mathrm{~N} \mathrm{ha}^{-1}$. A fonte de nitrogênio mineral foi sulfato de amônio. Como plantas testemunha não fixadoras de nitrogênio foram testadas as invasoras encontradas nas pastagens (Cyperus esculentus L., Amaranthus viridis L. e Solanum paniculatum L.), com seus valores de $\delta{ }^{15} \mathrm{~N}$ de 4,8 ; 5,7; 5,8 respectivamente e de 4,4 nas pastagens de um modo geral.

Após o corte de uniformização, foi feito

Tabela I. Características químicas do solo ${ }^{1}$ em três pastagens. (Chemical characteristics of the soil into three pastures).

\begin{tabular}{lcccccc}
\hline Pasto & $\mathrm{pH}^{2}$ & $\mathrm{~K}^{3}$ & $\mathrm{Ca}^{3}$ & $\mathrm{Mg}^{3}$ & $\mathrm{Al}^{3}$ & $\mathrm{P}^{4}$ \\
\hline B. decumbens & 5,4 & 1,7 & 3,7 & 1,3 & 0,2 & 21 \\
B. humidicola & 4,8 & 0,6 & 6,1 & 1,8 & 1,3 & 4 \\
P. purpureum & 5,5 & 0,5 & 6,1 & 1,8 & 0,0 & 8 \\
\hline
\end{tabular}

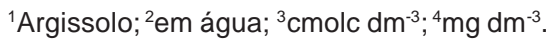


uma adubação uniforme de $\mathrm{P}$ ( 60 kg/ha) e K (100 kg/ha) nas pastagens, e a adubação nitrogenada nos tratamentos com N. O primeiro corte feito em fevereiro de 2001 correspondeu ao crescimento das plantas no período de transição seca/água. Os demais cortes foram realizados em abril/ 2001 (segundo), julho/2001 (terceiro) e agosto/2001 (quarto).

A técnica da abundância natural de ${ }^{15} \mathrm{~N}$ $\left(\delta^{15} \mathrm{~N}\right)$ tem como um de seus princípios, a utilização de uma testemunha não fixadora que possua um valor de delta semelhante ao do solo, pois nutrindo-se somente do $\mathrm{N}$ existente no solo, seu valor de delta será semelhante ou igual ao do mesmo. Esta testemunha pode ser qualquer planta não fixadora de nitrogênio que esteja ocorrendo espontaneamente na área de estudo.

A contribuição da FBN associada às pastagens foi estimada utilizando-se a técnica da abundância natural de ${ }^{15} \mathrm{~N}$ (Shearer e Kohl, 1986), em espectrômetro de massa Finnigan Mat, modelo Delta plus na Embrapa Agrobiologia. De acordo com esta técnica, a contribuição percentual de nitrogênio derivado da FBN, para a pastagem foi calculada através da fórmula:

$\% F B N=\left(\frac{\left(\delta^{15} \mathrm{~N} \text { planta testemunha }-\delta{ }^{15} \mathrm{~N} \text { planta teste) }\right.}{\left(\delta^{15} \mathrm{~N} \text { planta testemunha }-\mathrm{B}\right)}\right) \times 100$ Onde:

$\delta^{15} \mathrm{~N}$ da planta testemunha: valor de $\delta^{15} \mathrm{~N}$ do solo obtido através de plantas não fixadoras, utilizadas como referência;

$\delta^{15} \mathrm{~N}$ da planta teste: valor de $\delta^{15} \mathrm{~N}$ da planta fixadora de $\mathrm{N}_{2}$ (pastagem)

$\mathrm{B}$ : valor da discriminação isotópica de ${ }^{15} \mathrm{~N}$ feita pelas plantas durante o processo de FBN. Em plantas não nodulantes, o fracionamento isotópico, parece não ser da mesma magnitude que em plantas nodulantes, portanto para gramíneas, o valor B da fórmula geral foi considerado 0 (zero), conforme trabalho efetuado por Yoneyama et al. (1997).

Foi determinado o peso da matéria seca (PMS) de $1 \mathrm{~m}^{2}$ da parcela para estimativa da produção de cada pastagem. As amostras de plantas foram colocadas em estufa a $65^{\circ} \mathrm{C}$ durante 72 horas para determinação da matéria seca. A determinação do teor de nitrogênio total foi realizada pelo método Kjeldahl seguindo as etapas de digestão, destilação e titulação (Bremmer, 1965).

A contagem de microrganismos diazotróficos para determinação da população de bactérias expressa em $n^{\circ}$ de células/ml, foi realizada através da estimativa do Número Mais Provável (NMP) usando a tabela de MacCrady em meio semi-sólido NFB (Azospirillum spp.), JNFB (Herbaspirillum spp.) e LGI-P (Gluconacetobacter sp.) de acordo com a metodologia descrita por Döbereiner et al.(1995). A época de avaliação da população de bactérias foi em abril/2001 (início do período de chuva). A contagem de bactérias foi realizada apenas na época de chuvas onde o número sempre é maior do que na seca, assim sendio mais representativa, porém não foi realizado o isolamento de bactérias dos meios semi-sólidos.

As raízes foram coletadas diretamente no campo para contagem de bactérias diazotróficas e levadas para o laboratório de Biologia do Solo do IPA, Recife. Para contagem de diazotróficos em meio NFB (Azospirillum spp.) as raízes foram tratadas com água destilada/esterelizada, já para as bactérias endofíticas (Herbaspirillum spp. e Gluconacetobacter diazotrophicus) as raízes foram tratadas com Cloramina-T 1\% para esterilização da superfície das raízes, as amostras foram tratadas usando-se $10 \mathrm{~g}$ de raízes em um volume total de $100 \mathrm{ml}$ de solução salina. A contagem foi realizada nas diluições de $10^{-1}$ a $10^{-9}$ de acordo com metodologia de Döbereiner et al. (1995).

A análise estatística foi realizada utilizando-se o pacote estatístico MSTAT-C, da Universidade de Michigan, USA, onde foi utilizado o teste de Tukey a 5\% de probabilidade para separação de médias.

\section{RESULTADOSEDISCUSSÃO}

De maneira geral, a produção de matéria seca-MS (tabela II), das pastagens durante

Archivos de zootecnia vol. 59, núm. 225, p. 24. 
o período seco foi equivalente a $27 \%$ da forragem produzida no período das águas, o que é comum nas regiões tropicais. Este mesmo valor foi obtido por Souto (1982), nas condições do município de Itaguaí- RJ, durante as observações na produtividade de matéria seca de cinco gramíneas forrageiras (Hyparrhenia rufa cv. Deodoro, Digitaria decumbens cvs. Pangola A-21 e Transvala e Pennisetum purpurem cvs. Napier e Cameron), nas épocas, das águas e seca.

Apesar dos dados relativos à produção de MS no corte de transição seca-águas e no total dos cortes na época das águas não serem comparáveis estatísticamente entre as três pastagens, os valores obtidos para $P$. purpureum foram superiores aos de

Tabela II. Produção de matéria seca na parte aérea das forrageiras nas pastagens cultivadas com diferentes alturas de corte em $\mathrm{kg} M S / \mathrm{ha}$. (Dry matter production in the aerial part of the cultivate pastures with different cutting heights)

\begin{tabular}{lccccc}
\hline \multicolumn{5}{c}{ Transição } & \multicolumn{3}{c}{ Época das águas } \\
& fev & abr & jul & ago & Total \\
\hline B. decumbens & & & & & \\
$5 \mathrm{~cm}$ & $33,0^{\mathrm{a}}$ & $31,2^{\mathrm{a}}$ & $62,4^{\mathrm{a}}$ & $17,2^{\mathrm{a}}$ & $143,8^{\mathrm{a}}$ \\
$15 \mathrm{~cm}$ & $13,4^{\mathrm{b}}$ & $11,0^{\mathrm{b}}$ & $48,2^{\mathrm{a}}$ & $9,6^{\mathrm{b}}$ & $82,2^{\mathrm{b}}$ \\
$25 \mathrm{~cm}$ & $15,2^{\mathrm{b}}$ & $13,2^{\mathrm{b}}$ & $37,6^{\mathrm{a}}$ & $5,8^{\mathrm{b}}$ & $71,8^{\mathrm{b}}$ \\
$25 \mathrm{~cm}+\mathrm{N}$ & $10,8^{\mathrm{b}}$ & $9,8^{\mathrm{b}}$ & $28,0^{\mathrm{a}}$ & $9,6^{\mathrm{b}}$ & $58,2^{\mathrm{b}}$ \\
B. humidicola a & & & & & \\
$5 \mathrm{~cm}$ & $16,6^{\mathrm{ab}}$ & $11,0^{\mathrm{a}}$ & $8,4^{\mathrm{a}}$ & $28,2^{\mathrm{a}}$ & $64,2^{\mathrm{a}}$ \\
$15 \mathrm{~cm}$ & $13,0^{\mathrm{ab}}$ & $4,6^{\mathrm{b}}$ & $15,2^{\mathrm{a}}$ & $28,6^{\mathrm{a}}$ & $61,4^{\mathrm{a}}$ \\
$25 \mathrm{~cm}$ & $10,2^{\mathrm{b}}$ & $4,4^{\mathrm{b}}$ & $7,4^{\mathrm{a}}$ & $27,0^{\mathrm{a}}$ & $49,0^{\mathrm{a}}$ \\
$25 \mathrm{~cm}+\mathrm{N}$ & $17,8^{\mathrm{a}}$ & $5,0^{\mathrm{b}}$ & $14,6^{\mathrm{a}}$ & $24,6^{\mathrm{a}}$ & $62,0^{\mathrm{a}}$ \\
$P$. purpureum & & & & & \\
$5 \mathrm{~cm}$ & $156,6^{\mathrm{ab}}$ & $90,0^{\mathrm{a}}$ & $69,6^{\mathrm{a}}$ & $53,2^{\mathrm{a}}$ & $369,4^{\mathrm{a}}$ \\
$15 \mathrm{~cm}$ & $180,0^{\mathrm{a}}$ & $92,2^{\mathrm{a}}$ & $62,8^{\mathrm{a}}$ & $55,4^{\mathrm{a}}$ & $390,4^{\mathrm{a}}$ \\
$50 \mathrm{~cm}$ & $77,0^{\mathrm{b}}$ & $68,2^{\mathrm{a}}$ & $60,2^{\mathrm{a}}$ & $76,8^{\mathrm{a}}$ & $282,2^{\mathrm{a}}$ \\
$50 \mathrm{~cm}+\mathrm{N}$ & $86,8^{\text {ab }}$ & $69,0^{\mathrm{a}}$ & $63,2^{\mathrm{a}}$ & $53,4^{\mathrm{a}}$ & $272,4^{\mathrm{a}}$
\end{tabular}

fev: fevereiro; abr: abril; jul: julho; ago: agosto (2001). Médias seguidas de mesma letra nas colunas, para cada espécie e por época de avaliação, não diferem, entre si, Tukey $5 \%$.
Brachiaria decumbens e B. humidicola, no corte de transição seca-águas, e também no total dos cortes da época das águas. Esse comportamento de produção é expressão da capacidade genética do Pennisetum em produzir grande quantidade de matéria seca dependendo apenas de condições climáticas, de solo e do seu manejo (Souto, 1982; Fagundes et al., 2005).

A produção de MS de $B$. decumbens, no total dos cortes das duas épocas e no corte feito na transição seca-água, foi significativamente maior na intensidade de corte $5 \mathrm{~cm}$. É importante observar que o crescimento prostrado de B. decumbens, pode ter permitido que a forrageira tolerasse cortes mais drásticos, pois nas plantas dessas espécies, o meristema de crescimento encontra-se mais rente ao solo, possibilitando maior proteção nos cortes drásticos. No entanto, o manejo mais drástico de uma forrageira, seja por meio de maior intensidade ou maior freqüência de corte, com o tempo, afeta negativamente a sua persistência no pasto, principalmente nas pastagens sem a manutenção de adubação (Ortega e González, 1990; Lizieire et al., 1994). Lizieire et al. (1994), sugeriram que em experimentos de manejo com forrageiras, as observações devem abranger um período mais longo de duração.

Nas pastagens com $B$. humidicola e $P$. purpureum não foram encontradas diferenças estatísticas entre as intensidades de cortes na produção de MS total das duas épocas e nos cortes feitos na época das águas, exceto na avaliação da produção de MS na $B$. humidicola em abril/2001 e no corte na transição seca-águas, quando respectivamente, a intensidade de corte $5 \mathrm{~cm}$ foi superior as demais e o tratamento $25 \mathrm{~cm}+\mathrm{N}$ foi maior que o $25 \mathrm{~cm}$, evidenciando nesse caso, a eficiência da adubação nitrogenada na produção de MS da forrageira. Efeitos positivos da aplicação de $\mathrm{N}$ na produção de MS em capins forrageiros são encontrados na literatura (Martuscello et al., 2004; Gomide et al., 2006; Reis et al., 2007). Entre-

Archivos de zootecnia vol. 59, núm. 225, p. 25. 
tanto, não foram observadas diferenças estatísticas do efeito de $\mathrm{N}$ nos outros dois pastos, quando se comparou os tratamentos $25 \mathrm{~cm}$ e $25 \mathrm{~cm}+\mathrm{N}$ em B. decumbens e $50 \mathrm{~cm}$ $+50 \mathrm{~N}$ em $P$. purpureum. A falta de resposta desses pastos à adubação nitrogenda pode ser devido a lixiviação do N (Souto, 1982) aliada ao tempo decorrido entre à aplicação do adubo e avaliação de seu efeito nas plantas (Souto e Döbereiner, 1985). Na avaliação feita na transição seca-águas a produção de MS de $P$. purpureum na intensidade de corte $25 \mathrm{~cm}$ foi maior que 50 cm (tabela II).

Não foram observadas diferenças estatísticas entre as intensidades de cortes no acúmulo de $\mathrm{N}$ nas plantas de $B$. decumbens e $P$. purpureum nas avaliações feitas na época das águas, nos cortes feitos em $B$. humidicola em julho e agosto de 2001 e nas duas épocas para as três pastagens (tabela III). No entanto, na avaliação feita na transição seca-águas (fev/2001), a intensidade de corte $5 \mathrm{~cm}$ proporcionou maior acúmulo de $\mathrm{N}$ nas plantas de $B$. decumbens, conforme já havia sido observado para esta espécie na produção de MS, enquanto a intensidade 25 cm só foi diferente estatisticamente, da altura de corte $50 \mathrm{~cm}+\mathrm{N}$, no acúmulo de $\mathrm{N}$ nas plantas de $P$. purpureum.

Em relação aos efeitos dos tratamentos de intensidade de cortes no acúmulo de $\mathrm{N}$ na avaliação seca-águas nas plantas de $B$. humidicola, só foram encontradas diferenças significativas entre a altura de corte $5 \mathrm{~cm}$ e 25 cm, enquanto na $1^{\mathrm{a}}$ avaliação (abril/2001) na época das águas, o acúmulo de $\mathrm{N}$ nas plantas dessa espécie, a intensidade de corte 15 cm só foi significativamente superior a intensidade $25 \mathrm{~cm}$ (tabela III). Conforme já havia sido observado para produção de MS, também não foi observado resposta para os tratamentos com adubação nitrogenada, no acúmulo de $\mathrm{N}$ nas plantas das três pastagens. A adubação nitrogenada no presente experimento foi aplicada por ocasião do corte de uniformização, aproximadamente cinco meses antes do primeiro corte.
Na tabela IV, observa-se que os tratamentos fertilizados com $80 \mathrm{~kg} \mathrm{~N} / \mathrm{ha}$ na pastagem de $P$. purpureum, a população de bactérias diazotróficas testada (Azospirillum spp e G. diazotrophicus) foi inibida pelo nitrogênio aplicado na forma de sulfato de amônio, apesar do mesmo não ter interferido na produção de matéria seca e acúmulo do $\mathrm{N}$ nesssa gramínea, pois as bactérias diazotróficas são sensíveis à presença de nitrogênio mineral no sistema (Souto, 1982). Foram detectadas bactérias do gênero Azospirillum spp. na intensidade de corte a 25 cm de altura. Já a espécie G. diazotrophicus foi encontrada em todas alturas de corte. Quesada (2001) observou valores na população de bactérias em raízes de $P$. purpurem, 45,0 x $10^{5}$ células/ml para

Tabela III. Acúmulo de nitrogênio na parte aérea das forrageiras nas pastagens cultivadas com diferentes alturas de corte em $\mathrm{kg} \mathrm{N} /$ ha. (Accumulation of nitrogen in aerial parts of the cultivate forages with different cutting heights in $\mathrm{kg} \mathrm{N} / \mathrm{ha}$ ).

\begin{tabular}{lccccc}
\hline \multicolumn{5}{c}{ Transição } \\
fev & abr Época das águas \\
& & & jul & ago & Total \\
\hline B. decumbens & & & & & \\
$5 \mathrm{~cm}$ & $3,2^{\mathrm{a}}$ & $13,2^{\mathrm{a}}$ & $8,2^{\mathrm{a}}$ & $2,1^{\mathrm{a}}$ & $26,7^{\mathrm{a}}$ \\
$15 \mathrm{~cm}$ & $1,5^{\mathrm{b}}$ & $7,2^{\mathrm{a}}$ & $6,2^{\mathrm{a}}$ & $1,5^{\mathrm{a}}$ & $16,4^{\mathrm{a}}$ \\
$25 \mathrm{~cm}$ & $1,4^{\mathrm{b}}$ & $7,7^{\mathrm{a}}$ & $5,9^{\mathrm{a}}$ & $1,0^{\mathrm{a}}$ & $16,0^{\mathrm{a}}$ \\
$25 \mathrm{~cm}+\mathrm{N}$ & $0,8^{\mathrm{b}}$ & $5,6^{\mathrm{a}}$ & $3,8^{\mathrm{a}}$ & $1,3^{\mathrm{a}}$ & $11,5^{\mathrm{a}}$ \\
B. humidicola & & & & & \\
$5 \mathrm{~cm}$ & $11,5^{\mathrm{a}}$ & $1,4^{\mathrm{ab}}$ & $1,0^{\mathrm{a}}$ & $3,6^{\mathrm{a}}$ & $17,5^{\mathrm{a}}$ \\
$15 \mathrm{~cm}$ & $0,9^{\mathrm{ab}}$ & $2,1^{\mathrm{a}}$ & $2,3^{\mathrm{a}}$ & $3,7^{\mathrm{a}}$ & $9,0^{\mathrm{a}}$ \\
$25 \mathrm{~cm}$ & $0,8^{\mathrm{b}}$ & $0,9 \mathrm{~b}$ & $1,0^{\mathrm{a}}$ & $3,6^{\mathrm{a}}$ & $6,3^{\mathrm{a}}$ \\
$25 \mathrm{~cm}+\mathrm{N}$ & $1,2^{\mathrm{ab}}$ & $1,7^{\mathrm{ab}}$ & $2,2^{\mathrm{a}}$ & $3,5^{\mathrm{a}}$ & $8,6^{\mathrm{a}}$ \\
$P$. purpureum & & & & & \\
$5 \mathrm{~cm}$ & $11,5^{\mathrm{ab}}$ & $18,7^{\mathrm{a}}$ & $11,1^{\mathrm{a}}$ & $7,6^{\mathrm{a}}$ & $48,9^{\mathrm{a}}$ \\
$25 \mathrm{~cm}$ & $18,5^{\mathrm{a}}$ & $15,6^{\mathrm{a}}$ & $11,6^{\mathrm{a}}$ & $9,3^{\mathrm{a}}$ & $55,0^{\mathrm{a}}$ \\
$50 \mathrm{~cm}$ & $9,5^{\mathrm{ab}}$ & $15,4^{\mathrm{a}}$ & $10,4^{\mathrm{a}}$ & $12,8^{\mathrm{a}}$ & $48,1^{\mathrm{a}}$ \\
$50 \mathrm{~cm}+\mathrm{N}$ & $7,3^{\mathrm{b}}$ & $16,7^{\mathrm{a}}$ & $9,7^{\mathrm{a}}$ & $8,3^{\mathrm{a}}$ & $42,0^{\mathrm{a}}$ \\
\hline
\end{tabular}

fev: fevereiro; abr: abril; jul: julho; ago: agosto (2001). Médias seguidas de mesma letra nas colunas, para cada espécie e por época de avaliação, não diferem, entre si, Tukey $5 \%$. 
Tabela IV. População de bactérias diazotróficas (log do $n^{o}$ de células $/ \mathrm{ml}$ ) nas plantas das pastagens cultivadas com diferentes alturas de corte. (Diazothrophic bacteria populations in log of the cell number $/ \mathrm{ml}$ ).

\begin{tabular}{lccc}
\hline & \multicolumn{3}{c}{ Época das águas } \\
& Azosp & Herbasp & Gdiazot \\
\hline B. decumbens & & & \\
$5 \mathrm{~cm}$ & 6,4 & 5,4 & $\mathrm{Nt}$ \\
$15 \mathrm{~cm}$ & 6,4 & 5,4 & $\mathrm{Nt}$ \\
$25 \mathrm{~cm}$ & 6,4 & 0,0 & $\mathrm{Nt}$ \\
$25 \mathrm{~cm}+\mathrm{N}$ & $\mathrm{Nt}^{\star}$ & $\mathrm{Nt}$ & $\mathrm{Nt}$ \\
$B$. humidicola & & & \\
$5 \mathrm{~cm}$ & $\mathrm{Nd}$ & 7,1 & $\mathrm{Nt}$ \\
$15 \mathrm{~cm}$ & $\mathrm{Nd}$ & 5,4 & $\mathrm{Nt}$ \\
$25 \mathrm{~cm}$ & $\mathrm{Nd}$ & 5,4 & $\mathrm{Nt}$ \\
$25 \mathrm{~cm}+\mathrm{N}$ & $\mathrm{Nt}$ & $\mathrm{Nt}$ & $\mathrm{Nt}$ \\
$P$. purpureum & & & \\
$5 \mathrm{~cm}$ & $\mathrm{Nd}$ & $\mathrm{Nd}$ & 6,3 \\
$25 \mathrm{~cm}$ & 4,4 & $\mathrm{Nd}$ & 3,73 \\
$50 \mathrm{~cm}$ & $\mathrm{Nd}$ & $\mathrm{Nd}$ & 5,53 \\
$50 \mathrm{~cm}+\mathrm{N}$ & $\mathrm{Nd}$ & $\mathrm{Nt}$ & $\mathrm{Nd}$ \\
\hline
\end{tabular}

Azosp: Azospirilum spp.; Herbasp: Herbaspirillum spp. Gdiazot: G. diazotrophicus. $\mathrm{Nt}=$ não testado; $\mathrm{Nd}=$ não detectado.

Herbaspirillum spp. e 22,7 x $10^{5}$ células/ml para G. diazotrophicus.

Foi detectada a presença do gênero Azospirillum spp. nas raízes de $B$. decumbens nas intensidades de corte a 5, 15 e 25 cm de altura. Já o gênero Herbaspirillum spp. foi detectado quando as plantas foram submetidas aos cortes a 5 e $15 \mathrm{~cm}$ de altura (tabela IV).

A pastagem cultivada com $B$. humidicola apresentou, nos testes pelo NMP, valores não detectados para o gênero Azospirillum spp.(meio semi-sólido NFB), porém, o teste foi positivo para o gênero Herbaspirillum spp. (meio semi-sólido JNFB), com população de $12,5 \times 10^{6}$ células/ml na intensidade de corte a $5 \mathrm{~cm}$ de altura e $25,1 \times 10^{4}$ células/ml nas plantas cortadas a $15 \mathrm{~cm}$ e $25 \mathrm{~cm}$ de altura.

As testemunhas testadas para quantificação da FBN foram coletadas nas pastagens, sendo escolhida para quantificação a planta com maior valor de delta ${ }^{15} \mathrm{~N}$ (S. paniculatum. L.), e portanto, um método direto de avaliação, mas apresentando limitações nas interpretações em algumas situações (Watkins e Barraclough, 1996; Miranda, 2004). A planta testemunha deve ser oriunda de condições semelhantes às submetidas para as plantas testadas, pois ocorre variação espacial e temporal da abundância natural do isótopo ${ }^{15} \mathrm{~N}$ no solo, por haver também discriminação isotópica por parte da planta, esta é uma limitação da técnica isotópica em estudos para quantificação da contribuição da FBN em sistemas agrícolas. Porém, tomando-se o cuidado em avaliar o complexo que envolve o sistema de pastagem, como um todo, pode-se estimar valores quanto ao potencial da fixação biológica de nitrogênio, por este método (Boddey et al., 2000).

Quanto a contribuição da FBN (tabela V), não houve efeito significativo das intensidades de corte nas três pastagens. Souto e Takahashi (1989), encontraram também que as diferentes intensidades (15, 30 e 45 cm) e freqüências (6 e 12 semanas) de cortes não afetaram a FBN de $P$. purpureum cv. Cameron. No entanto, no período de transição da época seca/água, as pastagens de $B$. decumbens, $P$. purpureum e $B$. humidicola apresentaram na média das três intensidades de cortes, respectivamente, 32,4; 20,0 e 11,4\% do N fixado.

Na época das águas, a contribuição FBN foi mais expressiva nos meses de abril e julho de 2001, quando foram observados maiores índices de precipitação pluviométrica na região. Souto (1982) mostrou que a precipitação pluviométrica influenciou positivamente $\left(\mathrm{R}^{2}=0,76\right)$ naFBN decinco gramíneas forrageiras tropicais (Hyparrhenia rufa, Digitaria cvs. Pangola A-21 e Transvala e Pennisetum purpureum cvs. Napier $e$ Cameron).

Os resultados de Reis et al. (2001) relacionados a contribuíção da FBN de nove cultivares de $P$. purpureum, pela técnica de 
Tabela V. Contribuição da fixação biológica de nitrogênio (\%) das forrageiras nas pastagens cultivadas com diferentes alturas de corte. (Contribution (\%) of the biological nitrogen fixation of the cultivate forage pastures with different cutting heights).

\begin{tabular}{lcccc}
\hline \multicolumn{3}{c}{$\begin{array}{c}\text { Transição } \\
\text { fev }\end{array}$} & \multicolumn{3}{c}{ Época das águas } \\
abr & jul & ago \\
\hline B. decumbens & & & & \\
$5 \mathrm{~cm}$ & $31,6^{\mathrm{a}}$ & $42,2^{\mathrm{a}}$ & $23,6^{\mathrm{a}}$ & $9,4^{\mathrm{a}}$ \\
$15 \mathrm{~cm}$ & $29,6^{\mathrm{a}}$ & $40,8^{\mathrm{a}}$ & $33,0^{\mathrm{a}}$ & $14,6^{\mathrm{a}}$ \\
$25 \mathrm{~cm}$ & $36,0^{\mathrm{a}}$ & $39,2^{\mathrm{a}}$ & $22,6^{\mathrm{a}}$ & $10,4^{\mathrm{a}}$ \\
B. humidicola & & & & \\
$5 \mathrm{~cm}$ & $12,8^{\mathrm{a}}$ & $31,8^{\mathrm{a}}$ & $19,8^{\mathrm{a}}$ & $14,8^{\mathrm{a}}$ \\
$15 \mathrm{~cm}$ & $9,4^{\mathrm{a}}$ & $38,6^{\mathrm{a}}$ & $24,2^{\mathrm{a}}$ & $21,6^{\mathrm{a}}$ \\
$25 \mathrm{~cm}$ & $12,2^{\mathrm{a}}$ & $24,8^{\mathrm{a}}$ & $21,0^{\mathrm{a}}$ & $21,0^{\mathrm{a}}$ \\
P. purpureum & & & & \\
$5 \mathrm{~cm}$ & $18,4^{\mathrm{a}}$ & $26,4^{\mathrm{a}}$ & $23,0^{\mathrm{a}}$ & $13,0^{\mathrm{a}}$ \\
$25 \mathrm{~cm}$ & $20,2^{\mathrm{a}}$ & $25,8^{\mathrm{a}}$ & $24,6^{\mathrm{a}}$ & $13,6^{\mathrm{a}}$ \\
$50 \mathrm{~cm}$ & $22,6^{\mathrm{a}}$ & $22,0^{\mathrm{a}}$ & $32,0^{\mathrm{a}}$ & $21,2^{\mathrm{a}}$ \\
\hline
\end{tabular}

fev: fevereiro; abr: abril; jul: julho; ago: agosto (2001). Médias seguidas de mesma letra nas colunas, para cada espécie e por época de avaliação não diferem, entre si, Tukey $5 \%$.

diluição isotópica de de ${ }^{15} \mathrm{~N}$ mostraram que entre 25 e $40 \%$ de $\mathrm{N}$ da planta (105 e $165 \mathrm{~kg}$ $\mathrm{N} \mathrm{ha}^{-1}$ ) foram derivados da FBN, em uma amostragem no período seco em SeropédicaRJ. Em trabalho realizado por Quesada (2001), foi observada contribuição da FBN em $P$. purpureum de até $48 \%$, na época das águas. Souto e Silva (1989), sob condições

\section{BIBLIOGRAFIA}

Alves, B.J.R., Resende, A.S., Urquiaga, S. and Boddey, R.M. 2000. Biological nitrogen fixation by two tropical forage legumes assessed from relative ureide abundance of stem solutes ${ }^{15} \mathrm{~N}$ calibration of the technique in sand culture. Nutr. Cycl. Agroecosys., 56: 165-176.

Baldani, J.I. and Baldani, V.L.D. 2005. History on the biological nitrogen fixation research in graminaceous plants. Special emphasis on the Brasilian experience. An. Acad. Bras. Ciênc. de campo, encontraram que entre 13 capins selecionados para Baixada Fluminense, $B$. humidicola foi a espécie que apresentou maior FBN, em oito avaliações. Alves et al. (2000), em pastagem de B. humidicola mostraram também o ingresso total de $\mathrm{N}$, via FBN, de 84 e $56 \mathrm{~kg} \mathrm{~N} \mathrm{ha}^{-1}$, em taxas de lotação de 2 e 4 animais/ha, respectivamente. Boddey e Victoria (1986) por meio da técnica de diluição isotópica de de ${ }^{15} \mathrm{~N}$ registraram contribuíção de FBN para B. decumbens e $B$. humidicola, respectivamente de 40\% (40 kg $\mathrm{N} \mathrm{ha}^{-1}$ ) e $30 \%$ (30 $\left.\mathrm{kg} \mathrm{N} \mathrm{ha}^{-1}\right)$, enquanto Reis et al. (2001) usando a mesma técnica encontraram que a contribuíção da fixação biológica de $\mathrm{N}$ em plantas de $B$. decumbens e B. humidicola foram de 15,0 e 12,7\% e 19,4 e 9,7\%, respectivamente nos períodos, seco e das águas, no município de Seropédica-RJ.

\section{CONCLUSÕES}

Intensidade de corte mais drástica, proporcionou maior número de Azospirillum spp. e Herbaspirillum spp., maior produção de matéria seca e acúmulo de N-total em pastagens de $B$. decumbens na época de transição seca-águas, enquanto nas demais pastagens seus efeitos variaram com a época de avaliação.

A contribuição da fixação biológica de nitrogênio pelas bactérias diazotróficas nas pastagens variou de 10 a $42 \%$, mas as intensidades de corte não influenciaram na contribuição da fixação biológica de nitrogênio em nenhuma das pastagens.

77: 549-579.

Baldani, V.L.D., Baldani, J.I., Olivares, F.L. and Döbereiner, J. 1992. Identification and ecology of Herbaspirillum seropedicae and the closely H. rubrisubalbicans. Symbiosis, 13: 65-73.

Boddey, R.M., Peoples, M.B., Palmer, B. and Dart, P.J. 2000. Use of the $15 \mathrm{~N}$ natural abundance technique to quality biological nitrogen fixation by woody perennials. Nutr. Cycl. Agroecosys., 57: 235-270.

Archivos de zootecnia vol. 59, núm. 225, p. 28. 
Boddey, R.M., Jantalia, C.P., Macedo, M.O., Oliveira, O.C., Resende, A. S., Alves, B.J.R. and Urquiaga, S. 2006. Potential of carbon Atlantic forest region Brazil. In: Lal, R.; Cerri, C.C.; Bernoux, M.; Etchevers, J.; Cerri, E. (Eds.). Carbon sequestration in soil of Latin American. The Haworth Press. Binghamton. New York. pp. 305-347.

Boddey, R.M. and Victoria, R.L. 1986. Estimation of biological nitrogen-fixation associated with Brachiaria and Paspalum grasses using ${ }^{15} \mathrm{~N}$ labelled organic mattter and fertilizer. Plant Soil, 90: 265-294.

Bremmer, J.M. 1965. Inorganic nitrogen. In: D.P. Stewart (Ed.). Nitrogen fixation by free-living microorganisms. Cambridge Univ. Press. New York. 6: 249-258.

Döbereiner, J. 1992. History and new perspective of diazotrophs in association with nonleguminous plants. Symbiosis, 13: 1-13.

Döbereiner, J., Baldani, V.L.D. e Baldani, J.I. 1995. Como isolar e identificar bactérias diazotróficas de plantas não leguminosas. EMBRAPA, SPI. Itaguaí, RJ. EMBRAPA, CNPAB. Brasília. 60 pp.

Fagundes, J.L., Fonseca, D.M., Gomide, J.A., Nascimento Junior, S.D., Vitor, C.M.T., Morais, R.V., Mistura, C., Reis, G.C. e Martuscello, J.A 2005. Acúmulo de forragem em pasto de Brachiaria decumbens adubados com nitrogênio. Pesqui. Agropecu. Bras., 40: 397-403.

Gomide, C.A.M., Rangel, J.H.A., Jesus, R.S., Santos, N.L. e Silva, T.B. 2006. Resposta do capimMarandu a doses crescentes de nitrogênio ou do consórcio com Gliricidia sepium sob lotação rotacionada. Em: Reunião Anual da Sociedade Brasileira de Zootecnia, 41, Campo Grande. Anais. SBZ. Campo Grande.

Högberg, P., Högbom, L., Shinkel, H., Hörberg, M., Johannisson, C. and Wallmark, H. 1996. N-15 abundance of surface soils, roots and micorrhizas in profiles of European forest soils. Ecology, 108: 207-214.

Högberg, P. $1997 .{ }^{15} \mathrm{~N}$ natural abundance in soilplant systems. New Phitol., 137: 483-486.

Lizieire, R.S., Dias, P.F. e Souto, S.M. 1994. Efeito do manejo de cortes no desenvolvimento de forrageiras e nodulação de leguminosa. Em: Reunião Anual da Sociedade Brasileira de Zootecnia, 31, Campo Grande. Anais. SBZ. Maringá

Martuscello, J.A., Fonseca, D.M., Cunha, D.N.F.V., Moreira, L.M., Nascimento Junior, D., Santos,
P.M., Magalhães, M.A. e Santos, M.E.R. 2004. Perfilhamento da Brachiaria brizantha cv. Xaraés ob diferentes doses de nitrogênio e diferentes freqüências de corte. Em: Reunião Anual da Sociedade Brasileira de Zootecnia, 41. Campo Grande. Anais. SBZ. Campo Grande. Mattheus, C., Assuero, S.G., Black, C.K. and Sackville, H. 1999. Tiller dynamics of grazed swards. In: Congress on Grassland Ecophysiology and Grazing Ecology, Curitiba. Proceedings. UFPR/UFRGS. Curitiba. 109-133.

Miranda, C.H.B. 2004. As variações nos teores da abundância natural de ${ }^{15} \mathrm{~N}$ são boa indicação de fixação biológica de $\mathrm{N}^{2}$ em Brachiaria spp. In: Reunião Anual da Sociedade Brasileira de Zootecnia, Campo Grande. Anais. SBZ. Campo Grande.

Ortega, L.E. y González, B. 1990. Efecto de la fertilización nitrogenada y la frecuencia de corte sobre los rendimientos de materia seca y valor nutritivo del pasto estrella (Cynodon nlemfuensis). Rev. Agronomia, 7: 217-228.

Pena, K.S., Zanine, A.M., Silva, W.L., Sousa, B.M.L, Nascimento Junior, D. e Silva, S.C. 2007. Acúmulo de forragem de Panicum maximum cv. Tanzânia submetido a intensidades e freqüências de corte. Em: Reunião Anual da Sociedade Brasileira de Zootecnia, 44, Jaboticabal. Anais... SBZ. Jaboticabal.

Perin, R., Moraes, A. e Carvalho, P.C.F. 2006. Desempenho animal em uma consorciação de Panicum maximum Jacq. cv. Tanzânia com Arachis pintoi cv. Amarillo submetida a diferentes alturas de manejo. Em: Reunião Anual da Sociedade Brasileira de Zootecnia, 43. João Pessoa. Anais. SBZ. João Pessoa.

Quesada, D.M. 2001. Seleção de genótipos de capim elefante (Pennisetum purpureum Schum.) para a alta produção de biomassa e eficiência da fixação biológica de nitrogênio (FBN). 128 pp. Tese (Mestrado). Universidade Federal Rural do Rio de Janeiro. Seropédica.

Reis, G.H.C., Maranhão, C.M.A., Martins, G.C.F., Bonomo, P. e Pires, A.J.V. 2007. Avaliação do efeito da adubação nitrogenada e de intervalos de cortes da Brachiaria decumbens cv. Basilisk. Em: Reunião Anual da Sociedade Brasileira de Zootecnia, 44. Jaboticabal. Anais. SBZ. Jaboticabal.

Reis, V.M., Reis Jr., F.B., Quesada, D.M., Oliveira,

Archivos de zootecnia vol. 59, núm. 225, p. 29. 
O.C.A., Alves, B.J.R., Urquiaga, S. and Boddey, R.M. 2001. Biological nitrogen fixation associated with tropical pasture grasses. Austral. J. Plant Physiol., 28: 837-844.

Reis, V.M., Oliveira, A.L.M., Baldani, V.L.D., Olivares, F.L. e Baldani, J.I. 2006. Fixação biológica de nitrogênio simbiótica e associativa. Em: Fernandes, M.S. (Ed.). Nutrição mineral de plantas. Sociedade Brasileira de Ciência do Solo. Viçosa, 153-174.

Shearer, G.B. and Kohld, H. 1986. N2-fixation in field settings: estimations based on natural ${ }^{15} \mathrm{~N}$ abundance. Austral. J. Plant. Physiol., 13: 699756.

Sobrinho, F.S, Carneiro, H., Magalhães, J.R., Miranda, J.E.C., Pereira, A.V., Lédo, F.J.S., Reis M.C., Brum, S.S., Oliveira, J.S. e Botrel, M.A. 2005. Produtividade e qualidade da forragem de Brachiaria na região norte fluminense. Em: Reunião Anual da Sociedade Brasileira de Zootecnia, 42., Goiânia. Anais... SBZ. Goiânia.

Souto, S.M. 1971. Metodologia da introdução e avaliação de plantas forrageiras tropicais. Em: Dobereiner, J., Eira, P.A., Franco, A.A., Campelo, A.B. (Eds.). As leguminosas na agricultura tropical. DNPEA. Rio de Janeiro. 249-273.

Souto, S.M. 1982. Variação estacional da fixação de $\mathrm{N}_{2}$ e denitrificação em gramíneas forrageiras tropicais. 1982. Tese (Doutorado). Universidade Federal Rural do Rio de Janeiro. Seropédica. $268 \mathrm{pp}$.

Souto, S.M. e Döbereiner, J. 1985. Variação estacional da fixação de $\mathrm{N}_{2}$ e assimilação de nitrato em gramíneas forrageiras tropicais. Pesqui. Agropecu. Bras., 20: 319-334.

Souto, S.M., Franco, A.A. e Campello, E.F. 2003. Levantamento da ocorrência de árvores em pastagens em áreas de relevo acidentado no estado do Rio de Janeiro. Pasturas Tropicales, 25: 27-32.

Souto, S.M., Franco, A.A. e Campello, E.F. 2004. Espécies selecionadas para arborização das pastagens do estado do Rio de Janeiro. Pasturas Tropicales, 26: 1-47.

Souto, S.M. e Takahashi, N.T. 1989. Efeito do manejo de forrageiras na fixação biológica de nitrogênio. Em: Reunião Anual da Sociedade Brasileira de Zootecnia, 26, Porto Alegre. Anais. SBZ. Porto Alegre. p. 407

Souto, S.M. e Silva, T.P. 1989. Fixação biológica de nitrogênio na fase de estabelecimento de gramíneas forrageiras tropicais em um região do estado do Rio de Janeiro. Em: Reunião Anual da Sociedade Brasileira de Zootecnia, 26, Porto Alegre. Anais. SBZ. Porto Alegre. p. 408.

Tonato, F., Pedreira, C.G.S., Moreno, L.S.B. e Pedreira, B.C. 2007. Produtividade e distribuição estacional da produção de cinco gramíneas do gênero Cynodon sob manejo intensivo. Em: Reunião Anual da Sociedade Brasileira de Zootecnia, Jaboticabal. Anais. SBZ. Jaboticabal.

Urquiaga, S., Cruz, K.H.S. and Boddey, R.M. 1992. Contribution of nitrogen fixation to sugar cane: nitrogen-15 and nitrogen balance estimates. Soil Sci. Soc. An. J., 56: 105-114.

Watkins, N. and Barraclough, D. 1996. Gross rates of $\mathrm{N}$ mineralization associated with the decomposition of plant residues. Soil Biol. Biochem., 28: 169-175.

Yoneyama, T., Muraoka, T., Kim, T.H., Dacanay, E.V. and Nakanishi, Y. 1997. The natural ${ }^{15} \mathrm{~N}$ abundance of sugarcane and neighboring plants in Brazil, the Philippines and Miyako (Japan). Plant Soil, 189: 239-244.

Archivos de zootecnia vol. 59, núm. 225, p. 30. 